BeALs, R., and D. H. KRANTZ.

Math. Zeitschr. 101, 285-298 (1967)

\title{
Metrics and Geodesics Induced by Order Relations
}

\author{
Richard BeAls and DAVID H. Krantz *
}

Received December 27, 1966

\section{Introduction}

A metric on a space $S$ gives an order on $S \times S$, or equivalently, a mapping of $S \times S$ onto a totally ordered set $P$. Conversely, under suitable conditions such an order induces a uniformity on $S$ which is metrizable. This paper is concerned with the more delicate geometric question of what conditions such an order must satisfy to induce a "canonical" metric on $S$. By this we mean first that the order induced by the metric coincide with the original order, at least in the small. Second, the ternary relation $(x y z)$ on a metric space $(S, d)$, meaning that $d(x, y)+d(y, z)=d(x, z)$, can be given a plausible formulation in terms of the order on $S \times S$; we want $(x y z)$ to hold in this formulation if and only if it does with respect to the induced metric. More generally, a (geodesic) segment can be defined in terms of the relation $(x y z)$, and we want such a set to be a geodesic segment in the usual sense relative to the induced metric.

In terms of a mapping $(x, y) \rightarrow x y$ of $S \times S$ onto $P$, the conditions we impose are: (1) this mapping induces a non-discrete Hausdorff uniformity on $S$; (2) the mapping is continuous in each variable to the order topology of $P$; (3) $S$ is complete; (4) any point of $S$ can be reached from any other point in finitely many small steps; (5) a strong form of $M$-convexity holds. Precise formulation of these conditions is given in $\S 1$ and $\S 2$ below. Under these conditions, a metric $d$ which is canonical in the above sense exists and is essentially unique. Moreover, $S$ is arc-wise connected, and $(x y z)$ holds if and only if $y$ lies on a curve from $x$ to $z$ having length $d(x, z)$.

Applications of these results to the characterization of $G$-spaces in the sense of BUSEMANN [3] by order relations are given in $\$ 4$.

Several authors have investigated the connections between mappings from $S \times S$ to an ordered set and uniformities on $S$, e.g. APPERT [1] and the references there, KALISCH [5], and COHEN and GoFFMAN [4]; the question of introducing geometric entities, such as geodesic segments, in terms of orderings is not considered, however. The present work is more in the spirit of the investigations of the foundations of geometry, e.g. PIERI's axioms for Euclidean geometry (see [2]), which use only the concept " $y$ is equidistant from $x$ and $z$ ".

* The preparation of this paper was supported in part by the Air Force Office of Scientific Research Grant AF-AFOSR-736-65 and the National Science Foundation Grant NSF GP 5628 . 
The present results help to clarify the status of metric models in the behavioral sciences. In many empirical contexts one may hope to establish such qualitative relationships as " $x$ is more similar to $y$ than $z$ is to $w$ " in the absence of any quantitative measure of similarity (see the discussion in SHEPHARD [8]). We can now state qualitative conditions implying the existence of an $M$-convex metric compatible with such ordinal data. Similar results have been obtained previously in cardinal utility theory, where $S$ is one-dimensional, e.g. in SuPPES and WINET [9].

\section{Order and Uniformity}

Let $S$ be a non-empty set. Intuitively, a total order $\leqq$ on $S \times S$ should induce a uniformity on $S$ in which $x$ is at least as close to $y$ as $z$ is to $w$ if and only if $(x, y) \leqq(z, w)$. Rather than work directly with the order on $S \times S$, it is more convenient in what follows to work with a mapping from $S \times S$ to an ordered set $P$. The relations among orders, mappings, and non-discrete Hausdorff uniformities are sketched in this section.

We begin by making our terminology precise. An order on a set $A$ is a reflexive, transitive relation which we always denote by $\leqq$. We say that $A$ is strictly ordered if $a \leqq b$ and $b \leqq a$ imply $a=b$. We say that $A$ is totally ordered if for any $a, b \in A$, either $a \leqq b$ or $b \leqq a$. As usual we write $b \geqq a$ if $a \leqq b$, and $a<b$ if $a \leqq b$ but not $b \leqq a$.

If $A$ is a set and $U, V$ are subsets of $A \times A$, we let $U^{*}=\{(a, b) \mid(b, a) \in U\}$, and $U V=\{(a, c) \mid$ for some $b,(a, b) \in U$ and $(b, c) \in V\}$. We say $U$ is symmetric if $U^{*}=U$.

Let $S$ be a non-empty set, and $\leqq$ a total order on $S \times S$. Suppose that this order satisfies

$O_{1}$. For all $x, y, z \in S,(x, x) \leqq(y, z)$.

$O_{2}$. If $z \neq w$ and $x \in S$, then there is a $y \neq x$ with $(x, y)<(z, w)$.

$o_{3}$. For all $x, y \in S,(x, y) \leqq(y, x)$.

$O_{4}$. If $x \neq y$, there are $x^{\prime}, y^{\prime}$ with $x^{\prime} \neq y^{\prime}$, such that if $(u, v) \leqq\left(x^{\prime}, y^{\prime}\right)$ and $(v, w) \leqq\left(x^{\prime}, y^{\prime}\right)$, then $(u, w) \leqq(x, y)$.

Define an equivalence relation on $S \times S$ by $(x, y) \sim(z, w)$ if $(x, y) \leqq(z, w)$ and $(z, w) \leqq(x, y)$. Denote the equivalence class of $(x, y)$ by $x y$ and the set of such classes by $P$. Then $P$ is a strictly ordered, totally ordered set with minimal element 0 , and the mapping $(x, y) \rightarrow x y$ satisfies

$P_{1}, x y=0$ if and only if $x=y$.

$P_{2}$. If $a>0$ and $x \in S$, there is a $y$ with $0<x y<a$.

$P_{3}, x y=y x$.

$P_{4}$. If $b>0$, there is an $a>0$ such that $u v \leqq a$ and $v w \leqq a$ imply $u w \leqq b$.

Next, suppose $(P, \leqq)$ is a strictly ordered, totally ordered set with minimal element 0 , and suppose $(x, y) \rightarrow x y$ is a mapping from $S \times S$ onto $P$ which satisfies $P_{1}-P_{4}$. Given $a>0$, let $U_{a}=\{(x, y) \mid x y \leqq a\}$. Then $\underline{U}=\left\{U_{a}\right\}$ is a family of subsets of $S \times S$ which is totally ordered by inclusion and whose 
union is $S \times S$. Moreover,

$U_{1}$. $U=\Delta=\{(x, x) \mid x \in S\}$.

$U_{2}$. Given $x$ and $U$, there is a $y \neq x$ such that $(x, y) \in U$.

$U_{3}$. If $U \in \underline{U}, U=U^{*}$.

$U_{4}$. If $U \in \underline{U}$, there is a $U^{\prime} \in \underline{U}$ such that $U^{\prime} U^{\prime} \subseteq U$.

These are precisely the conditions that such a collection consist of symmetric sets which are a base for a non-discrete Hausdorff uniformity on $S$.

Suppose $\underline{U}$ is a family of subsets of $S \times S$ which is totally ordered by inclusion, whose union is $S \times S$, and which satisfies $U_{1}-U_{4}$. Given $x, y \in S$, let $U(x, y)$ be the intersection of all $U \in \underline{U}$ such that $(x, y) \in U$. Set $(x, y) \leqq(z, w)$ if $(x, y) \in U(z, w)$. Then it is not hard to see that $\leqq$ is a total order on $S \times S$ and $(S \times S, \leqq)$ satisfies $O_{1}-O_{4}$. For example, to prove $O_{4}$, suppose $x \neq y$. By $U_{1}$, there is a $U \in \underline{U}$ with $(x, y) \notin U$. Since $\underline{U}$ is totally ordered, it follows that $U \subseteq U(x, y)$. Take $U_{1} \in \underline{U}$ such that $U_{1} \cdot U_{1} \subseteq U$. Then let $x^{\prime}, y^{\prime}$ be such that $\left(x^{\prime}, y^{\prime}\right) \in U_{1}$ and $x^{\prime} \neq y^{\prime}$.

This gives a circle, order $\rightarrow$ mapping $\rightarrow$ totally ordered uniformity base $\rightarrow$ order. It is clear that if we start from an order (mapping) and proceed, we recover the same order (mapping). If we start from $U$, we return to $\{U(x, y)\}$, so two uniformity bases $\underline{U}$ and $\underline{V}$ induce the same order if and only if $U(x, y)=$ $V(x, y)$, all $x, y \in S$. Two such bases generate the same uniformity, for given $U \in U$, take $x \neq y,(x, y) \in U$. Take $V$ such that $(x, y) \notin V$. Then $V \subseteq V(x, y)=$ $U(x, y) \subseteq U$. Conversely, given $V \in V$ there is a $U \in \underline{U}$ with $U \subseteq V$.

The following property will also be useful; we give it in terms of the mapping.

$P_{5}$. If $b<c$, then there is an $a>0$ such that $x y \leqq b, y z \leqq a$ imply $x z<c$.

Remarks. 1. Condition $P_{4}$ follows from $P_{2}$ and $P_{5}$. For let $b>0$; by $P_{2}$, there is an $a_{1}$ with $0<a_{1}<b$. Choose $a_{2}>0$ such that $u v \leqq a_{1}, v w \leqq a_{2}$ imply $u w<b$. Then let $a=\min \left\{a_{1}, a_{2}\right\}$.

2. Condition $P_{5}$ is equivalent to the formally opposite property: if $b<c$ then there is an $a>0$ such that $x y \geqq c, z y \leqq a$ imply $x z>b$. For choose $a$ as in $P_{5}$. Then if $y z<a$ and $x z \leqq b$, we have $x y<c$; thus if $x y \geqq c$ and $y z<a$, necessarily $x z>b$. A similar argument shows that this property implies $P_{5}$.

Lemma 1.1. If $P_{1}-P_{5}$ hold, then for each $x \in S$ the mapping $y \rightarrow x y$ is continuous from the uniform topology of $S$ to the order topology of $P$.

This follows immediately from $P_{5}$ and Remark 2 .

Note that conversely if $P_{1}-P_{4}$ hold, if $y \rightarrow x y$ is continuous, and $P$ has no gaps, then $P_{5}$ holds.

\section{Convexity and Segments}

We assume from now on that $S$ is non-empty, $P$ is a strictly ordered, totally ordered set with minimal element 0 , and $(x, y) \rightarrow x y$ is a mapping of $S \times S$ onto $P$ satisfying $P_{1}-P_{5}$ of $\S 1$. Let $S$ have the induced uniform topology and 
$P$ the order topology. We now introduce three more conditions, significantly stronger than $P_{1}-P_{5}$.

$P_{6} . S$ is complete in the induced uniformity.

$P_{7}$. If $x, z \in S$ and $a>0$, there are points $y_{0}, y_{1}, \ldots, y_{n}$ in $S$ with $y_{0}=x$, $y_{n}=z$, and $y_{j-1} y_{j} \leqq a, j=1, \ldots, n$.

(Note that $P_{7}$ will necessarily be satisfied if $S$ is connected.)

The last condition, $P_{8}$, is a statement in terms of order of a strong from of $M$-convexity. Recall that in a metric space $(X, d)$ a ternary relation ( ) is defined by: $(x y z)$ if $x, y, z$ are distinct and $d(x, y)+d(y, z)=d(x, z)$. The space $X$ is said to be $M$-convex if for each $x \neq z$, there is a $y$ such that $(x y z)$; [3]. For $S$, we formulate these notions as follows.

Definition. The ternary relation \langle\rangle is defined in $S$ by: $\langle x y z\rangle$ if and only if $x, y, z$ are distinct and

(i) $x y^{\prime} \leqq x y$ and $x z^{\prime} \geqq x z$ imply $y^{\prime} z^{\prime} \geqq y z$;

(ii) If the conditions of (i) hold and $y^{\prime} z^{\prime}=y z$, then $x y^{\prime}=x y$ and $x z^{\prime}=x z$.

Definition. The ternary relation ( ) is defined in $S$ by: $(x y z)$ if and only if $\langle x y z\rangle$ and $\langle z y x\rangle$.

Remarks. 1. The relation ( $x y z)$ for metric spaces was introduced by MENGER [7].

2. In the case of a metric space $(X, d)$, let $x y=d(x, y)$. Then if $x, y, z$ are distinct, $d(x, y)+d(y, z)=d(x, z)$ implies that $(x y z)$ in the above sense. (The converse is false in general: consider the unit circle with the chordal metric.)

The final assumption is

$P_{8}$. If $0<x y^{\prime}<x z$, there is a $y \in S$ with $x y=x y^{\prime}$ and $(x y z)$.

We begin with some simple observations on the relation $(x y z)$.

Lemma 2.1. (a) If (xyz), then $x y<x z$ and $y z<x z$.

(b) If ( $x y z)$ and $x y^{\prime} \leqq x y, x z^{\prime} \geqq x z$, and $y^{\prime} z^{\prime} \leqq y z$, then $x y^{\prime}=x y, x z^{\prime}=x z$, $y^{\prime} z^{\prime}=y z$.

The proof is immediate.

Corollary 2.2. If $(x y z)$ and $x y^{\prime} \leqq x y, y^{\prime} z \leqq y z$, then $x y^{\prime}=x y, y^{\prime} z=y z$, and $\left(x y^{\prime} z\right)$.

Proof. Taking $z^{\prime}=z$ in Lemma 2.1(b), we get the desired equalities. Since $(x y z)$ it follows from these equalities and the definition that also $\left(x y^{\prime} z\right)$.

Lemma 2.3. If $(x y z)$ and $(x z w)$, then $(y z w)$ and $(x y w)$.

Proof. By Lemma 2.1(a), $x y<x z<x w$, so $y, z, w$ are distinct. Since ( $x y z)$ and $x z<x w, y z<y w$. Therefore there is a $z^{\prime}$ with $y z^{\prime}=y z$ and $\left(y z^{\prime} w\right)$. Then $z^{\prime} w \leqq z w$, so $x z^{\prime} \geqq x z$. But then, since $y z^{\prime}=y z$, we must have $x z^{\prime}=x z$, so $z^{\prime} w=z w$. Then by Corollary 2.2, $(y z w)$.

To show $(x y w)$ note that $y w<x w$, for otherwise $(x z w)$ would imply $y z \geqq x z$. Thus there is a $y^{\prime}$ with $y^{\prime} w=y w$ and $\left(x y^{\prime} w\right)$. By Corollary 2.2, it 
suffices to show that $x y \leqq x y^{\prime}$. Now $z w<y^{\prime} w$, so choose $z^{\prime}$ with $z^{\prime} w=z w$ and $\left(y^{\prime} z^{\prime} w\right)$. By the first part of this proof, $\left(x y^{\prime} z^{\prime}\right)$. But $(x z w)$ and $z^{\prime} w=z w$, so $x z \leqq x z^{\prime}$. If $x y^{\prime}<x y$, then $y z<y^{\prime} z^{\prime}$. But $\left(y^{\prime} z^{\prime} w\right), y^{\prime} w=y w$, and $z^{\prime} w=z w$, so $y z=y^{\prime} z^{\prime}$. Thus $x y^{\prime} \geqq x y$ and the proof is complete.

It is convenient to introduce an $n$-ary relation:

Definition. For $n \geqq 3,\left(x_{1} x_{2} \ldots x_{n}\right)$ if and only if for each $1 \leqq i<j<k \leqq n$, $\left(x_{i} x_{j} x_{k}\right)$.

Definition. Let $x$ and $z$ be distinct. A partial segment from $x$ to $z$ is a set $\gamma \subseteq S$ with the property that if $Y$ is a non-empty finite subset of $\gamma$ not containing $x$ or $z$, then there is an enumeration $y_{1}, \ldots, y_{n}$ of $Y$ such that $\left(x y_{1} \ldots y_{n} z\right)$. A segment is a maximal partial segment.

By ZoRN's lemma:

Proposition 2.4. Any partial segment from $x$ to $z$ is contained in a segment from $x$ to $z$.

The rest of this section is devoted to proving the following

Theorem 1. Let $x$ and $z$ be distinct points of $S$, and let $\gamma$ be a segment from $x$ to $z$. Then

(a) $\gamma$ is closed.

(b) The interval $[0, x z]=\{a \mid a \in P, 0 \leqq a \leqq x z\}$ is order complete, and for each $a \in[0, x z]$ there is a unique $y=y(a)$ in $\gamma$ such that $x y=a$.

(c) The mapping $a \rightarrow y(a)$ is a homeomorphism of $[0, x z]$ onto $\gamma$ with respect to the relative topology on $\gamma$.

The proof depends on several lemmas.

Lemma 2.5. If $\left(x_{1} x_{2} \ldots x_{n}\right)$ and $\left(x_{j} y x_{j+1}\right)$, then $\left(x_{1} \ldots x_{j} y x_{j+1} \ldots x_{n}\right)$.

Proof. Apply Lemma 2.3.

Lemma 2.6. If $a>0$ and $x \neq z$, there are $y_{0}, \ldots, y_{n}$ such that $y_{0}=x, y_{n}=z$, $\left(y_{0} \ldots y_{n}\right)$, and $y_{j-1} y_{j}<a, j=1,2, \ldots, n$.

Proof. By $P_{7}$ there are $u_{0}, \ldots, u_{p}$ with $u_{0}=x, u_{p}=z$, and $u_{j-1} u_{j}<a, j=$ $1, \ldots, p$. Let $y_{0}=u_{0}=x$. If $x z \leqq x u_{1}<a$, take $y_{1}=z$. Otherwise take $y_{1}$ such that $\left(x y_{1} z\right)$ and $y_{0} y_{1}=u_{0} u_{1}$. Then $y_{1} z \leqq u_{1} z$. Inductively, suppose $y_{0}, \ldots, y_{m}$ have been selected with $\left(y_{0} \ldots y_{m} z\right), y_{j-1} y_{j}<a$, and $y_{m} z \leqq u_{k} z$ for some $k \geqq m$. If $y_{m}=z$, we are done, and if $y_{m} z<a$, let $y_{m+1}=z$. Otherwise $y_{m} z \geqq a \geqq u_{p-1} z$, and take the largest $k$ with $m \leqq k \leqq p$ and $y_{m} z<u_{k} z$. Then $u_{k} u_{k+1}<a \leqq u_{k} z$, so there is a $v$ with $u_{k} u_{k+1}=u_{k} v$ and $\left(u_{k} v z\right)$. Then $v z \leqq u_{k+1} z<y_{m} z$, so there is $y_{m+1}$ with $y_{m+1} z=v z \leqq u_{k+1} z$ and $\left(y_{m} y_{m+1} z\right)$. Then $y_{m} y_{m+1} \leqq u_{k} v \leqq u_{k} u_{k+1}<a$, and $m+1 \leqq k+1$. Clearly this process terminates eventually with $y_{n}=z, n \leqq p$.

Let $P *$ denote the order completion of $P$.

Lemma 2.7. Let $\gamma$ be a partial segment from $x$ to $z$ and $a^{*} \in P^{*}$. If $\left\{y_{n}\right\} \subseteq \gamma$ is a generalized sequence such that $x y_{n} \rightarrow a^{*}$, then $\left\{y_{n}\right\}$ is a Cauchy generalized sequence. 
Proof. For all $n$, either $y_{n}=x$ or $y_{n}=z$ or $\left(x y_{n} z\right)$. Therefore $0 \leqq x y_{n} \leqq x z$, so $0 \leqq a^{*} \leqq x z$. If $a^{*}=0$, then $y_{n} \rightarrow x$. If $a^{*}=x z$, then given $a>0$, choose $y$ with $y z<a$ and $(x y z)$; (use $P_{2}$ and $P_{8}$ ). Then $x y<x z$, so for $n$ large, $x y_{n}>x y$. But either $y_{n}=z$ or $\left(x y_{n} z\right)$, so $y_{n} z<y z<a$. Thus $y_{n} \rightarrow z$.

Finally, suppose $0<a^{*}<x z$. Given $a>0$, we can use $P_{4}$ and Lemma 2.6 to find $u_{0}, u_{1}, \ldots, u_{m}$ such that $u_{0}=x, u_{m}=z,\left(u_{0} u_{1} \ldots u_{m}\right)$, and $u_{j-1} u_{j+1}<a$, $j=1, \ldots, m-1$. By Lemma 2.1(a), $0=x u_{0}<x u_{1}<\cdots<x u_{m}=x z$. Therefore for some $j, x u_{j-1}<a^{*}<x u_{j+1}$. Then for $n \geqq n_{0}, x u_{j-1}<x y_{n}<x u_{j+1}$. Then if $n, p \geqq n_{0}$, either $y_{n}=y_{p}$ or $x y_{n} \neq x y_{p}$. Suppose $x y_{n}<x y_{p}$. Then $x u_{j-1}<$ $x y_{n}<x y_{p}<x u_{j+1}$, and $\left(x y_{n} y_{p}\right)$, so $y_{n} y_{p}<u_{j-1} u_{j+1}<a$. Thus $\left\{y_{n}\right\}$ is Cauchy.

Lemma 2.8. If $y_{n} \rightarrow y$ and $\left(x y_{n} z\right)$, all $n$, then either $y=x$ or $y=z$ or $(x y z)$.

Proof. By Lemma 1.1, $x y=\lim x y_{n}$. Since $0<x y_{n}<x z$, all $n$, this implies $0 \leqq x y \leqq x z$. If $x y=0$, then $y=x$. If $x y=x z$, the first part of the preceding proof shows that $y_{n} \rightarrow z$, so $y=z$. Suppose $0<x y<x z$. Take $w$ such that $x w=x y$ and $(x w z)$. For $a>0$, choose $u_{a}$ such that $\left(x u_{a} w\right)$ and $u_{a} w<a$ (we use $P_{2}$ and $\left.P_{8}\right)$. Then $x u_{a}<x w=x y$, so for $n$ large, $x u_{a}<x y_{n}$. Since $\left(x y_{n} z\right)$, this implies $u_{a} z>y_{n} z$. Taking the limit in $n$, we get $u_{a} z \geqq y z$. As $a \rightarrow 0, u_{a} \rightarrow w$, so $w z \geqq y z$. Then Corollary 2.2 implies that $(x y z)$.

Lemma 2.9. Suppose $x \neq z$. A set $\gamma \subseteq S$ is a partial segment from $x$ to $z$ if and only if

(i) if $u \in \gamma$ and $u \neq x, u \neq z$, then ( $x u z)$;

(ii) if $u, v \in \gamma$ and $u, v, x$ are distinct, then ( $x u v)$ or $(x v u)$.

Proof. Necessity is obvious. Suppose conditions (i) and (ii) are satisfied. If $Y$ is a subset of $\gamma$ disjoint from $\{x, z\}$ and containing $n$ elements, we want to show that there is an enumeration $y_{1}, \ldots, y_{n}$ of $Y$ such that $\left(x y_{1} \ldots y_{n} z\right)$. If $n=1$, this follows from (i). Suppose it is true for $n \leqq p-1$. Then by (ii), for $u \neq v, u, v \in Y$, either $x u<x v$ or $x v<x u$. Number the elements of $Y$ with $x y_{1}<x y_{2}<\cdots<x y_{p}$. Now then by the induction assumption and these inequalities, $\left(x y_{2}, \ldots, y_{p} z\right)$. By (ii), $\left(x y_{1} y_{2}\right)$. Therefore, by Lemma 2.5, $\left(x y_{1} \ldots y_{p} z\right)$.

Proof of Theorem 1. (a) We suppose $\gamma$ is a segment from $x$ to $z$ and $\left\{y_{n}\right\}$ is a generalized sequence in $\gamma$ with $y_{n} \rightarrow y$. Since $\gamma$ is maximal, to show $y \in \gamma$ it suffices to show that $\gamma \cup\{y\}$ is a partial segment. We may assume $y \neq x, y \neq z$, and we use the criterion of Lemma 2.9. Lemma 2.8 implies that $(x y z)$, so it remains to show that if $u \in \gamma$ and $u, y, x, z$ are distinct, then $(x u y)$ or $(x y u)$. Now a simple modification of the argument in the first paragraph of the proof of Lemma 2.7 shows that if $x u=x y$, then $u=y$. Suppose $x u<x y$. For $n$ large, $x y_{n}>x u$, so $\left(u y_{n} z\right)$. By Lemma 2.8, then, $(u y z)$. But also $(x u z)$, so $(x u y)$. Similarly, if $x u>x y$ then $(x y u)$.

(b) Suppose $a^{*}$ is in the order completion of $[0, x z]$. We may assume $0<a^{*}<x z$. Let $\gamma_{1}=\left\{y \in \gamma \mid x y \leqq a^{*}\right\}, \quad \gamma_{2}=\left\{y \in \gamma \mid x y \geqq a^{*}\right\}$. Let $a_{1}^{*}=\sup \{x y \mid$ $\left.y \in \gamma_{1}\right\}$, in the order completion. Then there is a generalized sequence $\left\{y_{n}\right\} \subseteq \gamma_{1}$ such that $x y_{n} \rightarrow a_{1}^{*}$. By Lemma 2.7 and completeness, there is a $u_{1} \in S$ such 
that $y_{n} \rightarrow u_{1}$. By Lemma 1.1, $x u_{1}=a_{1}^{*}$, and by part (a) of this theorem, $u_{1} \in \gamma$. Similarly, if $a_{2}^{*}=\inf \left\{x y \mid y \in \gamma_{2}\right\}$, there is a $u_{2} \in \gamma$ such that $x u_{2}=a_{2}^{*}$. Thus either $a_{1}^{*}=a^{*}=a_{2}^{*}$ and $a^{*} \in P, x u_{1}=x u_{2}=a^{*}$, or else $a_{1}^{*}<a_{2}^{*}$. In the latter case, choose $u$ such that $\left(u_{1} u u_{2}\right)$. Then $u u_{2}<u_{1} u_{2}$, so since $\left(x u_{1} u_{2}\right)$ we have $x u_{1}<$ $x u_{2}$. Therefore $u \notin \gamma$. For any $y \in \gamma$, either $x y \leqq x u_{1}$ or $x y \geqq x u_{2}$. From this and Lemma 2.5 it follows easily that $\gamma \cup\{u\}$ is a partial segment. This contradicts maximality, so we must have $a_{1}^{*}=a^{*}=a_{2}^{*} \in P$, and $x u_{1}=x u_{2}=a^{*}$. Uniqueness follows from Lemma 2.1 (a).

(c) If $a_{n} \rightarrow a$ and $y_{n}=y\left(a_{n}\right), y=y(a)$, then $x y_{n}=a_{n} \rightarrow a$, so $\left\{y_{n}\right\}$ is Cauchy. Since $\gamma$ is closed, $u \rightarrow x u$ is continuous, $S$ is complete and $y(a)$ is unique, it follows that $y_{n} \rightarrow y$. Conversely, if $y_{n}=y\left(a_{n}\right), y=y(a)$, and $y_{n} \rightarrow y$, then $a_{n}=$ $x y_{n} \rightarrow x y=a$. Thus $a \rightarrow y(a)$ is a homeomorphism onto $\gamma$.

Corollary 1. If $\gamma$ is a segment from $x$ to $z$ and $u, v \in \gamma, u \neq v$, then $\gamma$ contains a unique segment from $u$ to $v$.

Proof. Let $\gamma^{\prime}=\{u, v\} \cup\{y \in \gamma \mid(u y v)\}$. This is a partial segment, so it is contained in a segment $\gamma^{\prime \prime}$. We may suppose $x u<x v$. Given $y \in \gamma^{\prime \prime}$, either $y=u$ or $y=v$ or $(u y v)$. Also, either $x=u$ or $(x u v)$. Therefore if $(u y v)$ then $(x y v)$, so there is a $y^{\prime} \in \gamma$ with $x y^{\prime}=x y$. Since $\left(x y^{\prime} v\right)$ also, we have $y^{\prime} v=y v>u v$, so $\left(u y^{\prime} v\right)$ and $u y^{\prime}=u y$. This shows that $y^{\prime} \in \gamma^{\prime} \subseteq \gamma^{\prime \prime}$, and it follows from $u y^{\prime}=u y$ that $y^{\prime}=y$. Thus $\gamma^{\prime}=\gamma^{\prime \prime}$ is a segment. Clearly any segment from $u$ to $v$ contained in $\gamma$ is contained in $\gamma^{\prime}$, hence is $\gamma^{\prime}$.

\section{Corollary 2. $P$ is connected.}

Proof. Since any interval $[0, a]$ in $P$ is order complete, $P$ is order complete. Therefore it is connected if and only if for each $a<c$, there is some $b$ with $a<b<c$; [6, p. 58]. Take $x, z$ such that $x z=c$. Let $\gamma$ be a segment from $x$ to $z$. Take $u \in \gamma$ such that $x u=a$. Now $\gamma$ contains a segment from $u$ to $z$, by Corollary 1. By $P_{2}$, there is an $a_{1}$ with $0<a_{1}<u z$, so there is a $v \in \gamma$ with $(u v z)$ and $u v=a_{1}$. Then $(x v z)$, so $a=x u<x v<x z=c$. Take $b=x v$.

Remarks. 1. Conditions $P_{1}-P_{4}$ and $P_{7}$ imply easily that $S$ is metrizable.

2. Conditions $P_{1}-P_{4}, P_{7}$ and $P_{8}$ imply that $P$ is separable (use $P_{4}$ and Lemma 2.6).

3. In view of the preceding remarks the arguments of this section could be carried out with ordinary sequences.

\section{Existence and Uniqueness of a Metric}

Let $S, P$, and the mapping $(x, y) \rightarrow x y$ satisfy conditions $P_{1}-P_{8}$. There is an essentially unique metric $|x, y|$ on $S$ which induces the given order on $S \times S$ in the small, and such that distinct points $x, y, z$ satisfy $(x y z)$ if and only if $|x, y|+|y, z|=|x, z|$. With respect to this metric, a segment from $x$ to $z$ is the image of a curve of length $|x, z|$, and conversely.

The plan of construction of this metric is the following. By "size" of a segment from $x$ to $z$ we mean the element $x z \in P$. For any positive integer $n$, 
there is a unique decomposition of a given segment into $n$ subsegments of equal size. For segments of size $a$ not too large, the size of these subsegments depends only on $a$ and $n$. These facts enable us to define an order isomorphism $a \rightarrow|a|$ of an interval $[0, \alpha)$ into the non-negative reals, with the property that (xyz) and $x z<\alpha$ imply $|x y|+|y z|=|x z|$. This mapping and the finiteness condition $P_{7}$ lead to a natural construction of $|x, y|$.

Lemma 3.1. Let $\gamma$ be a segment from $x$ to $z$ and let $n$ be a positive integer. Then there are unique points $y_{0}, \ldots, y_{n}$ in $\gamma$ such that $y_{0}=x, y_{n}=z,\left(y_{0} y_{1} \ldots y_{n}\right)$, and $y_{0} y_{1}=\cdots=y_{n-1} y_{n}$.

Proof. Given any ordered subset $Y=\left(u_{0}, \ldots, u_{n}\right)$ of $\gamma$ with $u_{0}=x, u_{1}=z$, and $\left(u_{0} u_{1} \ldots u_{n}\right)$, let $a(Y)=\inf \left\{u_{j-1} u_{j}\right\}$. Let $a$ be the supremum of $a(Y)$ for all such $Y$. Then $0 \leqq a \leqq x z$. Take $y_{0}=x$, and take $y_{1} \in \gamma$ such that $y_{0} y_{1}=a$. We can continue to choose $y_{j} \in \gamma$ with $\left(y_{0} y_{1} \ldots y_{p}\right)$ and $y_{j-1} y_{j}=a$ until either $y_{p} z<a$ for some $p<n$, or $y_{n-1} z>a$, or $y_{n-1} z=a$. In the third case, let $y_{n}=z$. We want to show the first two cases impossible.

Suppose $y_{p} z<a$, with $p<n$. Let $w_{p+1}=z$, and choose $w_{p} \in \gamma$ such that $\left(y_{p-1} w_{p} y_{p}\right)$. By making $w_{p} y_{p}$ small, we may assume $w_{p} z<a$. Continuing, we choose $w_{p-1}, \ldots, w_{1}$ with $w_{j-1} w_{j}<a$ and $\left(y_{j-1} w_{j} y_{j}\right)$. Let $w_{0}=y_{0}=x$. Let $a_{0}=\sup \left\{w_{j-1} w_{j}\right\}$. Then $a_{0}<a$. If $Y=\left(u_{0}, \ldots, u_{n}\right)$ is as above and $a(Y)>a_{0}$, inductively it is easy to see that $x u_{1}>x w_{1}, x u_{2}>x w_{2}, \ldots, x u_{p+1}>x w_{p+1}=x z$, a contradiction. Thus sup $a(Y) \leqq a_{0}<a$, a contradiction.

Suppose $y_{n-1} z>a$. Then we choose $u_{n}=z, u_{n-1} \in \gamma$ such that $\left(y_{n-1} u_{n-1} z\right)$ and $u_{n-1} z>a$, and $u_{n-2}, \ldots, u_{1}$ such that $\left(y_{j-1} u_{j-1} y_{j}\right)$ and $u_{j-1} u_{j}>a$. Let $u_{0}=x$ and $Y=\left(u_{0}, u_{1}, \ldots, u_{n}\right)$. Then $a(Y)=\inf \left\{u_{j-1} u_{j}\right\}>a$, a contradiction. This completes the proof of existence. Uniqueness follows by induction: $y_{j}=y_{j}^{\prime}$ and $y_{j} y_{j+1}=y_{j}^{\prime} y_{j+1}^{\prime}$ imply $y_{j+1}=y_{j+1}^{\prime}$, since all $y_{j}, y_{j}^{\prime}$ are in $\gamma$.

Definition. Given a segment $\gamma$ from $x$ to $z$ and a positive integer $n$, choose $y_{0}, \ldots, y_{n}$ as above. Let $y_{0} y_{1}=\cdots=y_{n-1} y_{n}=a(\gamma, n)$.

It is easy to see that

$$
a(\gamma, n+1)<a(\gamma, n), \quad \text { all } \gamma, n .
$$

Lemma 3.2. If $\gamma$ is a segment $x$ to $z$ and $a>0$, there is an $n$ such that $a(\gamma, n)<a$.

This follows from Lemma 2.6.

Lemma 3.3. Let $\gamma$ be a segment $x$ to $z$ and $\gamma^{\prime}$ be a segment from $x$ to $z^{\prime}$. For any $n, a(\gamma, n)<a\left(\gamma^{\prime}, n\right)$ if and only if $x z<x z^{\prime}$.

Proof. Divide each segment into $n$ equal parts and apply the definition of $(x y z)$ repeatedly.

In view of Lemma 3.3, $a(\gamma, n)$ depends only on $n$ and the endpoints $x, z$ of $\gamma$. We can define $a(x, z, n)=a(\gamma, n)$ for any segment $\gamma$ from $x$ to $z$. Then $a(x, z, n)<a\left(x, z^{\prime}, n\right)$ if and only if $x z<x z^{\prime}$. Set $a(x, x, n)=0$. 
Let

$$
\alpha=\sup _{x, z \in S}\{a(x, z, 6)\}
$$

if this set is bounded, and $\alpha=+\infty$, with $+\infty>a$, all $a \in P$, otherwise.

Lemma 3.4. Suppose $x, y \in S$ and $a<\alpha$. Then either $a(x, y, 2)>a$ or there is $a z \in S$ with both $a(x, z, 2)>a$ and $a(y, z, 2)>a$.

Proof. By definition of $\alpha$, there are $z_{0}, z_{1}, \ldots, z_{6}$ with $\left(z_{0} z_{1} \ldots z_{6}\right)$ and $z_{0} z_{1}=\cdots=z_{5} z_{6}>a$. Let $S_{0}=\left\{u \mid z_{0} u<z_{0} z_{2}\right\}$ and $S_{6}=\left\{u \mid z_{6} u<z_{6} z_{4}\right\}$. Then $S_{0}$ and $S_{6}$ are disjoint. If $x \notin S_{0}$ then $a\left(z_{0}, x, 2\right) \geqq a\left(z_{0}, z_{2}, 2\right)=z_{0} z_{1}>a$. Similarly for $y$ and for $S_{6}$. Thus either the second alternative holds, or else one of $x, y$ is in $S_{0}$ and the other in $S_{6}$. Assume $x \in S_{0}, y \in S_{6}$. Take $w$ such that $(x w y)$, $x w=w y=a(x, y, 2)$. Either $z_{0} w \geqq z_{0} z_{3}$ or $w z_{6} \geqq z_{3} z_{6}$. Thus either $a(x, y, 2)=$ $x w \geqq z_{2} z_{3}>a$ or $a(x, y, 2)=w y \geqq z_{3} z_{4}>a$.

This lemma enables us to prove a uniform property of the relation $(x y z)$.

Lemma 3.5. Suppose $(x y z)$ and $x z<\alpha$. If $x^{\prime} y^{\prime} \leqq x y$ and $x^{\prime} z^{\prime} \geqq x z$, then $y^{\prime} z^{\prime} \geqq y z$. Moreover, if $y^{\prime} z^{\prime}=y z$, then $x^{\prime} y^{\prime}=x y, x^{\prime} z^{\prime}=x z$, and $\left(x^{\prime} y^{\prime} z^{\prime}\right)$.

Proof. To prove the first statement, it suffices to consider the case $x^{\prime} y^{\prime}=$ $x y, x^{\prime} z^{\prime}=x z$. Suppose first that $a\left(x, x^{\prime}, 2\right)>x^{\prime} z^{\prime}=x z$. Let $\gamma$ be a segment from $x$ to $x^{\prime}$. We may assume that $y, y^{\prime}, z, z^{\prime}$ lie on $\gamma$. Then using Lemma 2.1 with respect to the midpoint of $\gamma$, we get $y^{\prime} z^{\prime}=y z$.

Next, if $a\left(x, x^{\prime}, 2\right)<x z=x z^{\prime}<\alpha$, then there is a point $u$ whose distance from $x$ and $x^{\prime}$ is greater than twice $x z$. Taking segments from $u$ to $x$ and to $x^{\prime}$, and using the first part of the proof to reflect about the midpoints of these segments, we again reduce to Lemma 2.1.

Finally, suppose $x^{\prime} y^{\prime} \leqq x y, x^{\prime} z^{\prime} \geqq x z$, and $y^{\prime} z^{\prime}=y z$. Choose $v$ such that $v=z^{\prime}$ or $\left(x^{\prime} v z^{\prime}\right)$ and $x^{\prime} v=x z$. Choose $u$ such that $\left(x^{\prime} u v\right)$ and $x^{\prime} u=x y$. Then by the first part, $u v \geqq y z$. But $y z=y^{\prime} z^{\prime} \geqq u v$. Thus $y^{\prime} z^{\prime}=u v$, so $x^{\prime} u=x^{\prime} y^{\prime}$ and $x^{\prime} z^{\prime}=x v$. But then $v=z^{\prime}$ and, by Corollary $2.2,\left(x^{\prime} y^{\prime} z^{\prime}\right)$.

As a consequence we have a significant extension of Lemma 3.3.

Corollary 3.6. If $x z<\alpha$ and $x^{\prime} z^{\prime}<\alpha$, then $a(x, z, n)<a\left(x^{\prime}, z^{\prime}, n\right)$ if and only if $x z<x^{\prime} z^{\prime}$.

Proof. Cf. Lemma 3.3.

Definition. If $0<a<\alpha$ and $n$ is a positive integer, then $(1 / n) a$ is the unique element of $P$ such that $(1 / n) a=a(x, y, n)$ when $x y=a$. (Uniqueness follows from Corollary 3.6.) Let $(1 / n) 0=0$.

From this definition and Corollary 3.6 we get

$$
\frac{1}{m}\left(\frac{1}{n} a\right)=\frac{1}{m n} a,
$$

$$
a<b \text { if and only if } \frac{1}{n} a<\frac{1}{n} b \text {. }
$$


Eq. (1) implies

$$
\frac{1}{n+1} a<\frac{1}{n} a \text { if } a>0 .
$$

We are now in a position to construct the desired metric.

Theorem 2. There is a metric $(x, y) \rightarrow|x, y|$ on $S$ with the properties

(i) For some fixed $\beta>0$, if $x y<\beta$ and $z w<\beta$, then $|x, y|<|z, w|$ if and only if $x y<z w$.

(ii) If $x, y$, and $z$ are distinct, then $(x y z)$ if and only if $|x, y|+|y, z|=|x, z|$.

(iii) For any $x, y, y^{\prime},|x, y|<\left|x, y^{\prime}\right|$ if and only if $x y<x y^{\prime}$. The properties (i) and (ii) determine $|x, y|$ uniquely, up to a multiplicative constant. Moreover, we may take $\beta=\alpha$.

Remark. A natural question is whether the restriction $x y<\alpha$ in (i) can be removed. An example in $\S 5$ shows that there may be $x, y, z, w$ with $x y<z w$ but $|x, y|>|z, w|$.

One way of achieving the conclusion $x y \leqq z w$ if and only if $|x, y| \leqq|z, w|$ is suggested by Lemma 3.5, which is the key to the proof of (i). For distinct points $x, y, z$, define $\langle x y z\rangle$ if and only if: $x^{\prime} y^{\prime} \leqq x y$ and $x^{\prime} z^{\prime} \geqq x z$ imply $y^{\prime} z^{\prime} \geqq y z$, and moreover if $y^{\prime} z^{\prime}=y z$ then $x^{\prime} y^{\prime}=x y$ and $x^{\prime} z^{\prime}=x z$. Define $((x y z))$ if and only if $\langle x y z\rangle$ and $\langle z y x\rangle$. Let $P_{8}^{\prime}$ be condition $P_{8}$ with $(())$ replacing ( ). Then the development in $\S 2$ is substantially the same, and Lemma 3.5 is true without restriction on the size of $x z$. Thus $P_{1}-P_{7}$ and $P_{8}^{\prime}$ imply that $x y \leqq z w$ if and only if $|x, y| \leqq|z, w|$.

Proof of Theorem 2. We begin with the last statement. Suppose $|x, y|$ is a metric satisfying (ii) and with $x y<\beta$ and $z w<\beta$ implying $|x, y|<|z, w|$ if and only if $x y<z w$. We may assume $\beta \leqq \alpha$. Then we can define a mapping of $[0, \beta)=\{a \mid 0 \leqq a<\beta\}$ into the reals unambiguously by letting $|a|=|x, y|$ if $x y=a<\beta$. Given any $x, z$, by Lemma 2.5 there are $y_{0}, y_{1}, \ldots, y_{n}$ with $y_{0}=x$, $y_{n}=z, y_{j-1} y_{j}<\beta$, and $\left(y_{0} y_{1} \ldots y_{n}\right)$. Then repeated application of (ii) gives $|x, z|=\sum\left|y_{j-1}, y_{j}\right|=\sum\left|y_{j-1} y_{j}\right|$. Thus $|x, z|$ is determined completely by the mapping $a \rightarrow|a|$ defined on $[0, \beta)$.

Note that (ii) implies that if $a<\beta$, then

$$
\left|\frac{1}{n} a\right|=\frac{1}{n}|a| .
$$

Choose $a_{0}$ such that $0<a_{0}<\beta$. We may assume that $\left|a_{0}\right|=1$. Note that if $a, b \in(0, \alpha)$, then it follows from Lemma 3.2 that there is an $m$ such that

$$
\frac{1}{m} a \leqq b \text {. }
$$

In particular, for each $n$ and each $a \in[0, \beta)$ there is a smallest $m=m(a, n)$ such that

$$
\frac{1}{m} a \leqq 2^{-n} a_{0}
$$


For $n$ large, $m(a, n)>1$. Then for $m=m(a, n)>1$, (i) implies

$$
m^{-1}|a| \leqq 2^{-n} \leqq(m-1)^{-1}|a|
$$

Furthermore, (2) and (3) imply $(2 m)^{-1} a \leqq 2^{-n-1} a<(2 m-2)^{-1} a$, so

$$
2 m(a, n)-1 \leqq m(a, n+1) \leqq 2 m(a, n)
$$

Let $|a|_{n}=2^{-n} m(a, n)$. Then (5) and (6) become

$$
\begin{aligned}
& |a|_{n}-2^{-n} \leqq|a| \leqq|a|_{n}, \\
& |a|_{n}-2^{-n} \leqq|a|_{n+1} \leqq|a|_{n} .
\end{aligned}
$$

It follows from (7) that $|a|_{n} \rightarrow a$. Thus $a \rightarrow|a|$ is completely determined by the condition $\left|a_{0}\right|=1$, and the final statement is proved.

To prove existence, take $a_{0} \in(0, \alpha)$. Given $a \in[0, \alpha)$, define $m(a, n)$ and $|a|_{n}$ as above. By (8), $\left\{|a|_{n}\right\}$ is a Cauchy sequence. Let $|a|=\lim |a|_{n}$. Given $(x y z)$ and $x z<\alpha$, let $\gamma$ be a segment from $x$ to $z$ containing $y$. Note that $m(x y, n)=m$ if and only if it takes $m-1$ steps along $\gamma$ of "size" $2^{-n} a_{0}$ to come within $2^{-n} a_{0}$ of $y$, starting at $x$. Therefore

$$
[m(x y, n)-1]+[m(y z, n)-1]<m(x z, n) \leqq m(x y, n)+m(x z, n),
$$

or

$$
|x y|_{n}+|y z|_{n}-2^{-n} \leqq|x z|_{n} \leqq|x y|_{n}+|x z|_{n} .
$$

Thus

$$
|x y|+|y z|=|x z|, \quad \text { if }(x y z) \text { and } x z<\alpha .
$$

Given $x, z$, take $|x, z|=0$ if $x=z$. Otherwise, let $\gamma$ be a segment from $x$ to $z$. By an admissible partition of $\gamma$ we mean an ordered subset $Y=\left(y_{0}, y_{1}, \ldots, y_{n}\right)$ of $\gamma$ such that $y_{0}=x, y_{n}=z,\left(y_{0} y_{1} \ldots y_{n}\right)$ and $y_{j-1} y_{j}<\alpha$. There is always an admissible partition with $n=7, y_{j-1} y_{j} \equiv a(\gamma, 7)$. If $Y$ and $Y^{\prime}=\left(y_{0}^{\prime}, \ldots, y_{m}^{\prime}\right)$ are admissible partitions of $\gamma$ with $Y \subseteq Y^{\prime}$, it is easy to see that repeated applications of (11) imply that $\sum\left|y_{j-1} y_{j}\right|=\sum\left|y_{j-1}^{\prime} y_{j}^{\prime}\right|$. Since any two partitions of $\gamma$ are contained in a single partition, this sum is independent of $Y$. By Lemma 2.3, it depends only on $x$ and $z$. Set $|x, z|=\sum\left|y_{j-1} y_{j}\right|$ for any such $Y$. In particular, if $x z<\alpha$ then $|x, z|=|x z|$, and (i) is satisfied. Furthermore, Lemma 3.3 implies that (iii) is satisfied.

It remains to be proved that $|x, z|$ is a metric and that it satisfies (ii). Suppose $(x y z)$. Let $\gamma$ be a segment containing $y$ (by Proposition 2.4), and let $Y$ be an admissible partition of $\gamma$ containing $y$. By Corollary 1 of Theorem $1, \gamma=\gamma_{1} \cup \gamma_{2}$ with $\gamma_{1}$ a segment from $x$ to $y$ and $\gamma_{2}$ a segment from $y$ to $z$. Similarly $Y=$ $Y_{1} \cup Y_{2}$ with $Y_{i}$ an admissible partition of $\gamma_{i}$. Then by definition of the metric we have $|x, y|+|y, z|=|x, z|$. This proves half of (ii).

Finally, let $x, y, z$ be any three points of $S$. If $x, y, z$ are not distinct, or if $x y \geqq x z$, then clearly $|x, y|+|y, z| \geqq|x, z|$. Suppose $0<x y<x z$. Take $y^{\prime}$ such 
that $\left(x y^{\prime} z\right)$ and $x y^{\prime}=x y$. Then $y^{\prime} z \leqq y z$, so by (iii),

$$
|x, z|=\left|x, y^{\prime}\right|+\left|y^{\prime}, z\right| \leqq|x, y|+|y, z| \text {. }
$$

Moreover, equality implies $y^{\prime} z=y z$, which implies $(x y z)$. This proves that $|x, y|$ is a metric and that the other half of condition (ii) is satisfied, completing the proof of Theorem 2 .

Theorem 3. If $x$ and $z$ are distinct points of $S$, and $\gamma$ is a segment from $x$ to $z$, there is a curve $\varphi$ from $x$ to $z$ with image $\gamma$ and length $|x, z| ; \varphi$ can be taken to be an isometry. Conversely, if $\varphi$ is a curve from $x$ to $z$ with length $|x, z|$, then its image is a segment from $x$ to $z$.

Proof. Let $y$ be a segment from $x$ to $z$. Let $a \rightarrow y(a)$ be the mapping of $[0, x z]$ onto $\gamma$ given in Theorem 1. Then $\eta: a \rightarrow|x, y(a)|$ is an order preserving map of $[0, x z]$ into $[0,|x, z|]$, hence is continuous. Since $P$ is connected, so is $[0, x z]$, and $\eta(0)=0, \eta(x z)=|x, z|$. Therefore $\eta$ is onto, and a homeomorphism. Thus $\varphi: t \rightarrow y\left(\eta^{-1}(t)\right)$ is a homeomorphism of $[0,|x, z|]$ onto $\gamma$. Suppose $0<s<t<|x, z|$. Then $|x, \varphi(s)|=s,|x, \varphi(t)|=t$, so $(x \varphi(s) \varphi(t))$ and $|\varphi(s), \varphi(t)|=|x, \varphi(t)|-|x, \varphi(s)|=|t-s|$. Thus $\varphi$ is an isometry, and its length is consequently $|x, z|$.

Conversely, suppose $\varphi$ is a curve from $x$ to $z$ with length $|x, z|$. Let $[0,1]$ be the domain of $\varphi$. For any partition $\left(t_{0}, \ldots, t_{n}\right)$ of $[0,1]$, we have

$$
|x, z| \geqq \sum\left|\varphi\left(t_{j-1}\right), \varphi\left(t_{j}\right)\right| \geqq|x, z| \text {. }
$$

It follows from Theorem 2 (ii) that if the $\varphi\left(t_{j}\right)$ are distinct, $\left(\varphi\left(t_{0}\right) \varphi\left(t_{1}\right) \ldots \varphi\left(t_{n}\right)\right)$. Thus the image $\gamma$ of $\varphi$ is a partial segment. Let $\gamma^{\prime}$ be a segment containing $\gamma$ and $\psi$ an isometry of $\gamma^{\prime}$ onto $[0,|x, z|]$ with $\psi(x)=0, \psi(z)=|x, z|$. Then $\psi \varphi$ maps $[0,1]$ continuously into $[0,|x, z|]$ with $\psi \varphi(0)=0, \psi \varphi(1)=|x, z|$. Therefore $\psi \varphi$ is onto, so $\varphi=\psi^{-1}(\psi \varphi)$ is onto $\gamma^{\prime}$, and $\gamma=\gamma^{\prime}$.

\section{On the Characterization of $\boldsymbol{G}$-Spaces by Order Relations}

As defined by BuSEMANN [3], a $G$-space is a metric space $(S,|x, y|)$ in which closed, bounded sets are compact and which satisfies the three additional conditions

A. If $x \neq z$, there is a $y$ with $(x y z)$.

B. For each $u$ there is an $a(u)>0$ such that if $x \neq y$ and $|x, u|<a(u)$, $|y, u|<a(u)$, then there is a $z$ with $(x y z)$.

C. If $(x y z),\left(x y z^{\prime}\right)$ and $|y, z|=\left|y, z^{\prime}\right|$, then $z=z^{\prime}$.

Here we take $(x y z)$ to mean $x, y, z$ are distinct and $|x, y|+|y, z|=|x, z|$.

We can easily translate the compactness condition and conditions $\mathrm{B}$ and $\mathrm{C}$ into terms of a mapping $(x, y) \rightarrow x y$ :

$P_{9}$. For $a \geqq 0$ and $x \in S$, the set $\{y \mid x y \leqq a\}$ is compact in the uniform topology. 
$P_{10}$. For $u \in S$ there is an $a(u)>0$ such that if $x u<a(u), y u<a(u)$, and $x \neq y$, then there is a $z$ such that $(x y z)$.

$P_{11}$. If $(x y z),\left(x y z^{\prime}\right)$, and $y z=y z^{\prime}$, then $z=z^{\prime}$.

As before, let $S$ be a non-empty set and $(x, y) \rightarrow x y$ a mapping of $S \times S$ onto an ordered set $P$ with minimal element 0 .

Theorem 4. Let $(S, x y)$ satisfy conditions $P_{1}-P_{5}$ and $P_{8}-P_{11}$. Then there is a metric $|x, y|$ on $S$ such that $(S,|x, y|)$ is a $G$-space and such that conditions (i) and (ii) of Theorem 2 are satisfied for some $\beta>0$. This metric is unique up to multiplicative constant.

Proof. Condition $P_{9}$ clearly implies $P_{6}$, completeness of $S$. Moreover $P_{1}-P_{5}, P_{8}, P_{9}$ imply $P_{7}$, the finiteness condition. For take $x, z \in S$ and $a>0$. We may assume $x z>a$. By $P_{8}$, there is a $y_{1}$ with $x y_{1}<a$ and $\left(x y_{1} z\right)$. If $y_{1} z<a$ we are through; otherwise take $y_{2}$ such that $x y_{1}=y_{1} y_{2},\left(y_{1} y_{2} z\right)$, and so on. Note that Lemmas 2.1 and 2.3 do not depend on $P_{6}$ and $P_{7}$. Thus we get $y_{0}=x, y_{1}, y_{2}, \ldots$ with $\left(x y_{1} y_{2} \ldots z\right)$ and $y_{0} y_{1}=y_{1} y_{2}=\ldots$. If $P_{7}$ did not hold, we would get an infinite sequence $\left\{y_{n}\right\}$, with $y_{m} y_{n} \geqq y_{m} y_{m+1}=y_{0} y_{1}$ for $n>m$, and $x y_{n}<x z$. This contradicts $P_{9}$.

Thus $P_{1}-P_{8}$ are all satisfied, and there is, up to a multiplicative constant, a unique metric $|x, y|$ on $S$ satisfying (i) and (ii) of Theorem 2. In view of (ii), the metric and order senses of $(x y z)$ coincide. Therefore $(S,|x, z|)$ satisfies A, B, C, by $P_{8}, P_{10}, P_{11}$ respectively. Thus $(S,|x, z|)$ is a $G$-space.

Conversely, let $(S,|x, y|)$ be a $G$-space. Let $P=\{|x, y|\}$ and $x y=|x, y|$. Then clearly $P_{1}-P_{5}$ are satisfied, as well as $P_{9}, P_{10}, P_{11}$. It follows from the general theory of $G$-spaces that $P_{8}$ is also satisfied. These observations and the preceding Theorem give

Corollary 1. If $(S,|x, y|)$ is a metric space, then there is, up to a multiplicative constant, at most one metric on $S$ which induces the same order on $S \times S$ and with respect to which $S$ is a G-space.

\section{Examples and Counter-Examples}

The following examples show that the conditions of completeness, finiteness, and convexity are independent, and that the order induced by the metric $|x, y|$ may not coincide globally with that induced by $x y$.

1. Let $S$ be the rationals, $P$ the non-negative rationals, and let the mapping be $(x, y) \rightarrow|x-y|$. Then conditions $P_{1}-P_{5}, P_{7}$, and $P_{8}$ are satisfied, but not $P_{6}$.

2. Let $S$ be $R \times R$ ( $R$ the reals) with the lexicographic order: $x=\left(x_{1}, x_{2}\right) \leqq$ $y=\left(y_{1}, y_{2}\right)$ if $x_{1} \leqq y_{1}$ or $x_{1}=y_{1}$ and $x_{2} \leqq y_{2}$. This is an ordered group with respect to addition. Let $P$ be the positive semi-group together with 0 , and let $x y=y-x$ if $y-x \geqq 0, x y=x-y$ otherwise. This satisfies $P_{1}-P_{8}$ except $P_{7}$; topologically it is an uncountable union of disjoint copies of $R$.

3. Let $S$ be the unit circle in $R \times R$, with an open arc of length less than $\pi$ removed. Let $P=[0,2]$ and let the mapping take a pair of points into their (plane) distance. This satisfies $P_{1}-P_{7}$, but not $P_{8}$. 
4. A more instructive example than (3) is the following. Let $S=R, P$ the non-negative reals, and let the mapping be $(x, y) \rightarrow g(|x-y|)$, where $g$ is any function on $P$ with $t \leqq g(t) \leqq 2 t$, all $t \geqq 0$. Then $P_{1}-P_{7}$ are satisfied. It can be seen that $P_{8}$ is satisfied if and only if $g$ is strictly increasing. If so, then we can take $|x, y|=|x-y|$.

5. Let $S=[0,2], P=\left[0, \frac{3}{2}\right]$. Define $g(x, r)$ for $x, r \in[0,2]$ by $g(x, r)=r$, $r \leqq 1$ and $g(x, r)=1+\frac{1}{2}(x+1)(r-1), r>1$. For $x, y \in S$ and $x \leqq y$, define $x \cdot y=$ $y \cdot x=g(x, y-x)$. We claim that the mapping $(x, y) \rightarrow x \cdot y$ is a metric, and that $P_{1}-P_{8}$ are satisfied. The key point is that the function $g(x, y-x)$ strictly decreases in $x$ for $0 \leqq x \leqq y$ and strictly increases in $y$ for $x \leqq y \leqq 2$. To show $P_{8}$, suppose for example that $x<z$ and $0<a<x \cdot z$. There is a unique $y$ with $x<y<z$ and $x \cdot y=a$. Suppose $x \cdot y^{\prime} \leqq x \cdot y$ and $x \cdot z^{\prime}=x \cdot z$. Because of the property of $g$ cited above, we may reduce to the case $z^{\prime}<y^{\prime}<x$ and $x \cdot y^{\prime}=$ $x \cdot y, x \cdot z^{\prime}=x \cdot z$. If $x \cdot z \leqq 1$, then all distances coincide with usual ones, so $y^{\prime} \cdot z^{\prime}=y \cdot z$. But $x \cdot z>1$ implies $z-x>1$ and, since $x \cdot z^{\prime}=x \cdot z$, also $x-z^{\prime}>1$. Then $z-z^{\prime}>2$, a contradiction. Thus $\langle x y z\rangle$, and similarly $\langle z y x\rangle$.

Now since $S$ satisfies $P_{1}-P_{8}$ and since $x \cdot y=|y-x|$ for $|y-x| \leqq 1$, it follows from Theorem 2 that we can take $|x, y|=|y-x|$, all $x, y \in S$. It is easy to see that there are points $x, y, z, w$ with $x \cdot y<z \cdot w$ but $|x-y|>|z-w|$; e.g., take $x=0, y=\frac{3}{2}, z=\frac{2}{3}, w=2$.

Remark. The example (5) is not a $G$-space in the induced metric, since segments cannot be prolonged past the endpoints. It would be interesting to know whether $P_{1}-P_{5}$ and $P_{8}-P_{11}$ imply that the original order and the induced order necessarily coincide globally. This is true in the non-compact case, and is also true when the induced $G$-space turns out to be an $n$-dimensional torus, for example.

\section{References}

[1] APPERT, A.: Écart partiellement ordonné et uniformité. C. R. Acad. Sci. (Paris) 224, $442-444(1947)$.

[2] BLumenthal, L. M.: Distance geometries: a study of the development of abstract metrics. Univ. of Missouri Studies 13, No. 2 (1938).

[3] BusemanN, H.: The geometry of geodesics. New York 1955.

[4] Cohen, L. W., and C. Goffman: On the metrization of uniform space. Proc. Amer. Math. Soc. 1, 750-753 (1950).

[5] KalisCH, G. K.: On uniform spaces and topological algebra. Bull. Amer. Math. Soc. 52, 936-939 (1946).

[6] KeLley, I. L.: General topology. Princeton 1955.

[7] MenGer, K.: Untersuchungen über allgemeine Metrik. Math. Ann. 100, 75-163 (1928).

[8] Shepard, R. N.: The analysis of proximities: multidimensional scaling with an unknown distance function, I. Psychometrika 27, 125-140 (1962).

[9] SUPPES, P., and M. WINET: An axiomatization of utility based on the notion of utility differences. Management Sci. 1, 259-270 (1955).

Department of Mathematics, University of Chicago, Chicago, Ill., U.S.A. and Department of Psychology, University of Michigan, Ann Arbor, Mich., U.S.A. 